\section{Rimonabanto: perspectivas e controvérsias}

Rimonabant: perspectives and controversies

\author{
Karine Zortéa, Rafaela Festugatto Tartari* \\ * Nutricionista.
}

\section{Caros Editores,}

Doenças cardiovasculares são consideradas um problema de saúde pública, sendo responsáveis por $30 \%$ das mortes no mundo ${ }^{1}$. Diabetes, hipertensão arterial, tabagismo e o surgimento de novos fatores de risco (aumento da circunferência abdominal e dislipidemia), contribuem reconhecidamente para o risco cardiovascular global ${ }^{2}$. A população adulta americana apresenta cerca de $66 \%$ de sobrepeso e $34 \%$ de obesidade ${ }^{3}$. No Brasil, a prevalência é de aproximadamente $32 \%$ de sobrepeso e $8 \%$ de obesidade $^{4}$. Conseqüentemente, especialistas têm desenvolvido novas condutas para tratar/prevenir essa condição.

Vem crescendo a atenção no potencial terapêutico das substâncias que interferem no sistema endocanabinóide ${ }^{5}$, devido ao seu papel no controle da ingestão alimentar e balanço energético. Este sistema age em áreas específicas do cérebro, como o hipotálamo, e em tecidos periféricos (adipócitos, hepatócitos, trato gastrointestinal e músculo esquelético). Portanto, drogas (rimonabanto) que interferem em sua ação são promissoras no tratamento de diversas doenças, incluindo a obesidade, dislipidemia, resistência à insulina e aterosclerose ${ }^{6}$. O rimonabanto tem aprovação no Brasil ${ }^{7}$ e na Europa, mas não nos Estados Unidos ${ }^{8}$. Grandes estudos comprovaram a eficácia do rimonabanto, como o RIO-Europe, RIO-North America, RIO-Lipids e RIO-Diabetes. Um ano de tratamento com rimonabanto $20 \mathrm{mg} /$ dia, juntamente com uma dieta hipocalórica e promoção de atividade física, produziram redução no peso, na circunferência da cintura, e melhoras no colesterol HDL, triglicerídeos, glicose e resistência à insulina. Apesar de o rimonabanto ser bem tolerado, pode apresentar efeitos colaterais como alterações no humor, depressão, ansiedade, tonturas, náuseas e, até mesmo, ideações suicidas 9 .

\footnotetext{
Nota dos Editores: Após o aceite desta carta, o rimonabanto foi retirado do mercado pela agência de regulação devido aos seus importantes efeitos colaterais. Apesar disso, decidimos manter a publicação, como demonstração de que tais comunicações dão suporte científico à prática clínica.
}

O Strategy to Reduce Atherosclerosis Development Involving Administration of Rimonabant - the Intravascular Ultrasound Study (STRADIVARIUS) é outro grande estudo, realizado na América do Norte, Europa e Austrália, onde pacientes com doença cardiovascular e obesidade abdominal receberam $20 \mathrm{mg}$ de rimonabanto, durante 18 meses. Houve redução no peso corporal, melhora no perfil lipídico, redução nos níveis de proteína C-reativa e na hemoglobina glicosilada. No entanto, também foram observados efeitos colaterais, como depressão, ansiedade $(43,4 \%)$ e casos de ideação suicida, sendo que um paciente em uso da medicação realmente suicidou-se ${ }^{10}$.

Os efeitos colaterais psiquiátricos foram a principal preocupação da Food and Drug Administration (FDA) contra a aprovação do rimonabanto em $2007^{8}$. Apesar de os estudos relatarem baixos índices de complicações, um quadro de depressão e de intenção suicida não pode ser considerado irrelevante. Se houveram casos como esses e, principalmente, após a comprovação de um caso real de suicídio, os profissionais devem ser alertados. Antes de ocorrer a prescrição desse medicamento, o paciente deve ser rigidamente avaliado em suas condições psicológicas, para que se evite um agravamento no quadro de depressão. Mesmo assim, corre-se o risco de um indivíduo psicologicamente normal desenvolver um quadro depressivo em função da medicação. Esse manuscrito visa a conscientização dos profissionais de saúde, com o intuito de prevenir o uso indiscriminado desse fármaco.

\section{Referências}

1. Strong K, Mathers C, Leeder S, Beaglehole R. Preventing chronic diseases: how many lives we can save? Lancet. 2005;366(9496):1578-82.

2. Cleeman JI, Grundy SM, Becker D, Clark LT, Cooper RS, Denke MA, et al. Executive Summary of the Third Report of the National Cholesterol Education Program (NCEP) Expert Panel on Detection, Evaluation, and Treatment of High Blood Cholesterol in Adults (Adult Treatment Panel III). JAMA. 2001;285(19):2486 -97.

3. Ogden CL, Carroll MD, Curtin LR, McDowell MA, Tabak CJ, Flegal KM. Prevalence of overweight and obesity in the United States, 1999-2004. JAMA. 2006;295(13):1549-55.

4. Sposito AC, Caramelli B, Fonseca FAH, Bertolami MC, Afiune NA, Souza AD, et al. IV Diretriz brasileira sobre dislipidemias e prevenção da aterosclerose: Departamento de Aterosclerose da Sociedade Brasileira de Cardiologia. Arq Bras Cardiol. 2007;88(S1):2-18.

5. Godoy-Matos AF, Guedes EP, Souza LL, Valério CM. O sistema endocanabinóide: novo paradigma no tratamento da síndrome metabólica. Arq Bras Endocrinol Metab. 2006;50(2):390-9.

6. Pagotto U, Marsicano G, Cota D, Lutz B, Pasquali R. The emerging role of the endocannabinoid system in endocrine regulation and energy balance. Endocr Rev. 2006;27(1):73-100. 
7. Sanofi-aventis Laboratório. Acomplia ${ }^{\circledR}$ (Rimonabanto) é aprovado no Brasil para o tratamento de pacientes obesos ou com sobrepeso com fatores de risco cardiometabólico associados como diabetes do tipo 2 ou dislipidemia. http://www.sanofiaventis.com.br/live/br/pt/layout.jsp?cnt=A6FED1FE-9C3D4E15-8161-F3FD54B6E606\&strSelectView=CONTENT NORMAL\&strPageView $=2$. Acessado jul 2008.

8. Rumsfeld JS, Nallamothu BK. The hope and fear of rimonabant. JAMA. 2008;299(13):1601-2.
9. Van Gaal L, Pi-Sunyer X, Després JP, McCarthy C, Scheen A. Efficacy and safety of rimonabant for improvement of multiple cardiometabolic risk factors in overweight/obese patients: pooled 1-year data from the Rimonabant in Obesity (RIO) program. Diabetes Care. 2008;31 Suppl 2:S229-40.

10. Nissen SE, Nicholls SJ, Wolski K, Rodés-Cabau J, Cannon CP, Deanfield JE, et al. Effect of rimonabant on progression of atherosclerosis in patients with abdominal obesity and coronary artery disease: the STRADIVARIUS randomized controlled trial. JAMA. 2008; 299(13):1547-60. 